\author{
П. В. НАЙДУ'
}

\title{
ШИРОКОПОЛОСНАЯ ЗЕРКАЛЬНАЯ Z- И L-ОБРАЗНАЯ УНИПЛАНАРНАЯ ТРЕХДИАПАЗОННАЯ АНТЕННА С АСS-ФИДЕРОМ ДЛЯ WLAN ПРИМЕНЕНИЙ
}

\author{
${ }^{1}$ Инженерный колледж Велагапуди Рамакришна Сиддхарта, \\ Индия, Виджаявада, 520007 \\ ${ }^{2}$ Институт технологии и инженерии им. Чанакья, \\ Индия, Джайпур, 302022
}

\begin{abstract}
Аннотация. В работе предложена зеркальная Z- и L-образная печатная унипланарная антенна, разработанная для трехдиапазонного применения. Разработанная геометрия реализует простые излучающие полоски с 50 Ом асимметричной копланарной полосковой ACS (Asymmetric Coplanar Strip) питающей линией и прямоугольной заземляющей плоскостью. Эта антенна имеет компактные размеры $16 \times 24$ мм, включая заземляющую плоскость, которая имеет размер $0,18 \times 0,34 \lambda$ на частоте 2,3 ГГц в свободном пространстве. Результаты моделирования, полученные с помощью пакета CST Microwave Studio, сравнивались с результатами измерений печатной унипланарной антенны при помощи векторного анализатора PNA N5222A, в результате чего достигнуто хорошее совпадение результатов. Ширина полосы рабочих частот при коэффициенте отражения -10 дБ составила приблизительно 230 МГц в диапазоне 2,27-2,5 ГГц, 200 МГц в диапазоне 3,65-3,85 ГГц и 1700 МГц в диапазоне 5,2-6,9 ГГц. Предложенная унипланарная антенна имеет не только компактные размеры, но и широкие полосы рабочих частот, позволяющие работать в сетях LTE (Long Term Evolution), беспроводных широкополосных сетях WiBro (Wireless Broadband), ceтях WiMAX (Worldwide Interoperability for Microwave Access), беспроводных локальных сетях WLAN (Wireless Local Area Network) и системах диапазона ISM (Industrial Scientific and Medical radio band).
\end{abstract}

Ключевые слова: компактная антенна; трехдиапазонная антенна; антенна с ACS-фидером; монопольная антенна; многодиапазонная антенна

\section{1. ВВЕДЕНИЕ}

Современные тенденции в сфере техники беспроводной связи предполагают повышенное внимание к использованию одной системы для нескольких применений. В СВЧ области это особенно касается проектирования и совершенствования антенн, которые обеспечивают возможность работы в нескольких диапазонах частот наряду с малыми размерами, широкой полосой частот, большим коэффициентом усиления и возможностями аккумулирования электромагнитной энергии, что играет важную роль в совершенствовании техники нынешнего и будущего поколений.

Для обеспечения указанных характеристик предложены различные конструкции антенн при использовании разных схем питания [1-24]. Среди предложенных конструкций антенна с ACS-фидером (Asymmetric Coplanar Strip) является наиболее привлекательной и представляет собой лучший императивный подход, который позволяет уменьшить размеры печатной унипланарной антенны приблизительно на $45 \%[1,2]$. Кроме того, многодиапазонная рабочая характеристика получена в виде 


\section{БИБЛИОГРАФИЧЕСКИЙ СПИСОК}

1. Deepu., V; Rohith, K. Raj; Manoj, Joseph; Suma, M. N.; Mohanan, P. "Compact asymmetric coplanar strip fed monopole antenna for multiband applications," IEEE Trans. Antennas Propag.," Vol. 55, No. 8, p. 2351-2357, 2007. DOI: $10.1109 /$ tap.2007.901847.

2. Naidu, P. V. "Printed V-shape ACS-fed compact dual band antenna for bluetooth, LTE and WLAN/WiMAX applications," Microsyst. Technol., Vol. 23, No. 4, p. 1005-1015, 2017. DOI: 10.1007/s00542-0162939-7.

3. Li, B.; Yan, Z.-H.; Zhang, T.-L. "Triple-band slot antenna with U-shaped open stub fed by asymmetric coplanar strip for WLAN/WiMAX applications," PIER Lett., Vol. 37, p. 123-131, 2013. DOI: 10.2528/pierl1212 2601.

4. Lin, C.-P.; Chang, C.-H.; Jou, C. F. "Compact quad band monopole antenna," Microwave Opt. Technol. Lett., Vol. 53, No. 6, p. 1272-1276, 2011. DOI: $\underline{10.100}$ 2/mop.25994.
5. Ren, W.; Hu, S.-W.; Jiang, C. "An ACS-fed F-shaped monopole antenna for GPS/WLAN/WiMAX applications," Int. J. Microwave Wireless Technologies, Vol. 9, No. 5, p. 1123-1129, 2017. DOI: $\underline{10.1017 / \mathrm{s} 175}$ 9078716001173.

6. Saad, Ayman Ayd R.; Ibrahim, Ahmed A.; Haraz, Osama M.; Elboushi, Ayman. "Tri-band compact ACS-fed meander-line antenna for wireless communications," Int. J. Microwave Wireless Technol., Vol. 9, No. 9, p. 1895-1903, 2017. DOI: 10.1017/s1759 078717000745 .

7. Kang, L.; Wang, H.; Wang, X. H.; Shi, X. "Compact ACS-fed monopole antenna with rectangular SRRs for tri-band operation," Electron. Lett., Vol. 50, No. 16, p. 1112-1114, 2014. DOI: 10.1049/el.2014.1771.

8. Li, X.; Shi, X.-W.; Hu, W.; Fei, P.; Yu, J.-F. "Compact triband ACS-fed monopole antenna employing open-ended slots for wireless communication," IEEE Antennas Wireless Propag. Lett., Vol. 12, p. 388-391, 2013. DOI: $10.1109 /$ lawp.2013.2252414

9. Ren, W.; Jiang, C.; Hu, S.-W. "An asymmetric coplanar strip-fed 7-shaped monopole antenna for miniaturized communication systems," Microw. Opt. Technol. Lett., Vol. 58, No. 7, p. 1566-1572, 2016. DOI: 10.1002/mop.29858.

10. Song, Yue; Jiao, Yong Chang; Wang, Xiao Ming; Weng, Zi Bin; Zhang, Fu Shun. "Compact coplanar slot antenna fed by asymmetric coplanar strip for $2.4 / 5 \mathrm{GHz}$ WLAN operations," Microwave Opt. Technol. Lett., Vol. 50, No. 12 , p. $3080-3083$, 2008. DOI: $10.1002 /$ mop.23 $\underline{882}$.

11. Deepu, V.; Sujith, R.; Mridula, S.; Aanandan, C. K.; Vasudevan, K.; Mohanan, P. "ACS fed printed F-shaped uniplanar antenna for dual band WLAN applications," Microwave Opt. Technol. Lett., Vol. 51, No. 8, p. 1852-1856, 2009. DOI: 10.1002/mop.24486.

12. Ren, X.-S.; Yin, Y.-Z.; Hu, W.; Wei, Y.-Q "Compact tri-band rectangular ring patch antenna with asymmetrical strips for WLAN/WiMAX applications," $J$. Electromagnetic Waves Applications, Vol. 24, No. 13, p. 1829-1838, 2010. DOI: $10.1163 / 156939310792486548$.

13. Naidu, P. V.; Malhotra, A. "A small ACS-fed tri-band antenna employing $\mathrm{C}$ and $\mathrm{L}$ shaped radiating branches for LTE/WLAN/WiMAX/ITU wireless communication applications," Analog Integr. Circ. Sig. Process., Vol. 85, No. 3, p. 489-496, 2015. DOI: 10.1007/s10470-015-0637-5.

14. Ashkarali, P.; Sreenath, S.; Sujith, R.; Dinesh, R.; Krishna, D. D.; Aanandan, C. K. "A compact asymmetric coplanar strip fed dual-band antenna for DCS/WLAN applications," Microwave Opt. Technol. Lett., Vol. 54, No. 4, p. 1087-1089, 2012. DOI: 10.1002/mop.26731.

15. Kumar, A.; Naidu, P. V. "A compact O-shaped printed ACS fed monopole dual-band antenna for $2.4 \mathrm{GHz}$ Bluetooth and 5GHz WLAN/WiMAX applications," Proc. of 2016 Progress in Electromagnetic Research Symp., 8-11 Aug. 2016, Shanghai, China. IEEE, 2016. DOI: $10.1109 /$ piers.2016.7734856. 
16. Kumar, R.; Naidu, V. P.; Kamble, V. “A compact asymmetric slot dual band antenna fed by CPW for PCS and UWB applications," Int. J. RF Microwave Computer-Aided Engineering, Vol. 25, No. 3, p. 243-254, 2015. DOI: $\underline{10.1002 / \mathrm{mmce} .20855}$.

17. Wu, Y.-J.; Sun, B.-H.; Li, J.-F.; Liu, Q.-Z. "Triple-band omni-directional antenna for WLAN application," PIER, Vol. 76, p. 477-484, 2007. DOI: $\underline{10.2528 / \text { pier07080601. }}$.

18. Hua, M. J.; Wang, P.; Zheng, Y.; Yuan, S. L. "Compact tri-band CPW-fed antenna for WLAN/WiMAX applications," Electron. Lett., Vol. 49, No. 18, p. 1118-1119, 2013. DOI: $\frac{10.1049 / \mathrm{el} .2013 .1669}{\text {. }}$.

19. Cui, Y.-Y.; Sun, Y.-Q.; Yang, H.-C.; Ruan, C.-L. "A new triple-band CPW-fed monopole antenna for WLAN and WiMAX applications," PIER M, Vol. 2, p. 141-151, 2008. DOI: 10.2528/PIERM08041004.

20. Zhao, G.; Zhang, F.-S.; Song, Y.; Weng, Z.-B.; Jiao, Y.-C. "Compact ring monopole antenna with double meander lines for $2.4 / 5 \mathrm{GHz}$ dual-band operation," PIER, Vol. 72, p. 187-194, 2007. DOI: 10.2528/pier07031405.

21. Thomas, K. G.; Sreenivasan, M. "Compact triple band antenna for WLAN/WiMAX applications," Electron. Lett., Vol. 45, No. 16, p. 811-813, 2009. DOI: 10.1049/el.2009.1658.

22. Wei, C.-Y.; Liu, J.-C.; Hung, T.-F.; Bor, S.-S.; Chen, C. C. "A compact size and small frequency ratio CPW-fed circular slot antenna for GPS/WLAN dual-band and circular polarizations," PIER C, Vol. 22, p. 1-9, 2011. DOI: $10.2528 /$ PIERC11050410.

23. Li, D.; Zhang, F.-S.; Zhao, Z.-N.; Ma, L.-T.; Li, X. N. "A compact CPW-fed Koch snowflake fractal antenna for WLAN/WiMAX applications," PIER C, Vol. 28, p. 143-153, 2012. DOI: 10.2528/pierc12022106.

24. Lee, J. N.; Kim, Ji H.; Park, Jong K.; Kim, Jin S. "Design of dual-band antenna with U-shaped open stub for WLAN/UWB applications," Microwave Opt. Technol. Lett., Vol. 51, No. 2, p. 284-289, 2009. DOI: 10.1002/ mop. 24033.

25. Kumar, A.; Naidu, P. V.; Kumar, V.; Ramasamy, A. K. "Design \& development of compact uniplanar semi-hexagonal ACS fed multi-band antenna for portable system application," PIER M, Vol. 60, p. 157-167, 2017. DOI: $10.2528 /$ PIERM17080302.
26. Kumar, A.; Naidu, P. V.; Kumar, V. “A compact uniplanar ACS fed multi band low cost printed antenna for modern 2.4/3.5/5 GHz applications," Microsyst. Technol., Vol. 24, No. 3, p. 1413, 2018. DOI: 10.1007/s00542-0173556-9.

27. Naidu, P. V.; Kumar, A. "Design and development of triple band ACS fed antenna with $\mathrm{M}$ and rectangular shaped radiating branches for $2.45 / 5 \mathrm{GHz}$ wireless applications," Microsyst. Technol., Vol. 23, No. 12, p. 5841-5848, 2017. DOI: 10.1007/s00542-017-3430-9.

28. Naidu, P. V.; Kumar, A. "A novel ACS fed multi band antenna loaded with mirrored $\mathrm{S}$ and $\mathrm{L}$ shaped strips for advanced portable wireless communication applications," Microsyst. Technol., Vol. 23, No. 10, p. 4775-4783, 2017. DOI: 10.1007/s00542-017-3313-0.

29. Naidu, P. V., \& Kumar, A. (2018). ACS-Fed e-Shaped Dual Band Uniplanar Printed Antenna for Modern Wireless Communication Applications. Radioelectronics and Communications Systems, 61(3), 87-93. doi:10.3103/s0735272718030019.

30. Naidu, P. V.; Malhotra, A. "Design \& analysis of miniaturized asymmetric coplanar strip fed antenna for multi-band WLAN/WiMAX applications," PIER C, Vol. 57, p. 159-171, 2015. DOI: 10.2528/pierc15042302.

31. Naidu, P. V.; Malhotra, A.; Kumar, R. "A compact ACS-fed dual-band monopole antenna for LTE, WLAN/WiMAX and public safety applications," Microsyst. Technol., Vol. 22, No. 5, p. 1021-1028, 2015. DOI: $10.1007 / \mathrm{s} 00542-015-2562-\mathrm{Z}$.

32. Naidu, P. V.; Kumar, R. "A very small asymmetric coplanar strip fed multi-band antenna for wireless communication applications," Microsyst. Technol., Vol. 22, No. 9, p. 2193-2200, 2015. DOI: $10.1007 / \mathrm{s} 00542-015-$ 2613-5.

33. Naidu, P. V.; Kumar, R. "Design of a compact ACS-fed dual band antenna for Bluetooth/WLAN and WiMAX applications," PIER C, Vol. 55, p. 63-72, 2014. DOI: $10.2528 /$ pierc14101803.

34. Dawar, P., De, A., \& Raghava, N. S. (2018). S-Shaped Metamaterial Ultra-Wideband Directive Patch Antenna. Radioelectronics and Communications Systems, 61(9), 394-405. Doi: 10.3103/s0735272718090029. 